\title{
Photoactivation of bacterial phytochromes studied by time-resolved crystallography
}

\author{
Sebastian Westenhoffa \\ ${ }^{a}$ Department of Chemistry and Molecular Biology, University of Gothenburg, Sweden, \\ westenho@chem.gu.se
}

Eye-less species use photosensor proteins to collect information about ambient light conditions. Phytochromes are a photosensor superfamily in plants, fungi, bacteria. Upon photoactivation of a biliverdin cofactor, the chromophore and protein undergo a series of structural changes on multiple time- and length scales in order alter the biochemical output activity. The structures of the resting and light-activated states of bacteriophytochromes are known, but the structural mechanism with which light cues are transferred into structural rearrangements are not well understood. In particular, the primary structural response of the chromophore and the surrounding residues remains elusive.

Here, we present a crystallographic investigation of the photoresponse the phytochrome from $D$. Radiodurans. We present a new room-temperature structure obtained by serial femtosecond X-ray crystallography at the Japanese X-ray free electron laser. I will also discuss time-resolved snapshots of the protein and its implication for the primary photoresponse of phytochrome proteins. 